Words go missing in cyberspace

Sir - Your Briefing on electronic journals asked "Why do researchers download and print?" rather than reading articles on the screen (Nature 397, 195-200; 1999). The author suggests that the explanation lies with the poor resolution of text displays on computer screens. I think the real problem lies much deeper, in the way our brains process information, and will not be susceptible to a simple technological fix.

Reading requires us to pile one level of abstraction on top of another. We must recognize letters from the shapes of marks on the page, and the meanings of words from those letters and from the sounds our minds associate with them, and the information in sentences from the meanings of words and from grammatical rules, and still-moreabstract concepts from the sentences. One feature of reading that makes this achievement possible is that it is grounded in a physical object, the page or book.

One example of the importance of physical context in reading is our positional memory for text - frequently we can easily remember the location of a piece of information on the page and in the book, even though we've forgotten its textual content and context. I suspect that this physical framework is a key part of our reading ability, especially for difficult material.

When we read online we forfeit this level of perception - all text is ephemeral. It flows like water across the screen, and appears and disappears with the click of a mouse. For serious reading of the scientific literature, an abstract may be all we can absorb from the screen without becoming disoriented. It's as if the ideas we're reading can't be given locations in our minds if they don't have locations in space.

\section{Rosie Redfield}

Department of Zoology,

University of British Columbia,

Vancouver, British Columbia V6T 1Z4, Canada

Sir - Your Briefing indicates that one promising model for Internet publishing is to ask authors to pay page (as well as figure and table) charges, so reducing the journal's costs. We see two serious flaws with this approach.

First, as long as there are journals that do not require page charges, journals that do so will compete at a serious disadvantage for quality manuscripts. Second, the charge per page will jeopardize the ability of an enormous body of authors - namely those from developing nations - to publish in such journals. This is all the more relevant because the Internet frees publishers to a large extent from the onerous page limit restrictions of the printed medium, greatly reducing costs.

We have opted in Ciencia al Día, a new electronic-only journal published in Spanish (and soon to be translated into English), to publish free of page charges and at no cost to the reader (http://www.ciencia.cl/CienciaAlDia/). This should help to narrow the South-toNorth knowledge gap discussed by Vanderlei Canhos and colleagues (Nature $397,201 ; 1999)$. In addition, the authors retain their copyrights and can publish the material elsewhere if they desire.

Jorge Golowasch ${ }^{\star}$, Tania Bedrax-Weiss $\dagger$, Adrián Palacios $¥$

(Editors, Ciencia al Día)

${ }^{*}$ Volen Center, Brandeis University, Waltham,

Massachusetts 02454, USA

$\dagger$ Computational Intelligence Research Laboratory, University of Oregon, Eugene,

Oregon 97403-1269, USA

$\$$ Departamento de Fisiología, Facultad de Ciencias, Universidad de Valparaiso, Valparaiso, Chile

\section{Whales, science and activism}

Sir - I hold no brief for Greenpeace, but those of us who monitor these things know that, far from taking action against the stricken whaling ship, as Dan Goodman asserts, the Greenpeace vessel, which happened to be nearby, offered assistance — which was refused - by both radio and signal flags (Nature 397, 290; 1999).

Rather than disregarding the advice of its scientific committee, as Goodman asserts, the International Whaling Commission formally accepted it, but has delayed implementation until the whaling nations agree to precautionary measures including independent international inspection and monitoring. What Goodman may find hard to accept is that scientific advice on possible safe catch quotas was nearly all based on research funded by environmental and animal welfare groups, including Greenpeace!

Some organizations in Japan apparently want to resolve the whaling issue. But the Japanese Institute of Cetacean Research, of which Goodman is a member, has made a mockery of its "scientific whaling programme" by resuming, more than half way through the summer season, the plan to catch the originally decided number of minke whales, despite the fact that this plan was said to have been drawn up to cover all of the designated "sampling" areas and seasons.

Sidney J. Holt

Podere il Falco, Ponticelli PG, 06060, Italy

Many a slip

\section{'twixt cup and lip}

Sir - I read with fascination Len Fisher's Commentary on the science and art of biscuit dunking (Nature 397, 469; 1999). Regrettably, I have not dunked for some years, as the outstanding biscuit for this enjoyment, the traditional English ginger nut, is rare in my country. Danish butter cookies are unsuitably crumbly. However, as a formerly avid dunker in England, I challenge his recommendation of nearhorizontal (NH) dunking.

This technique allows dunking only if you abstain from drinking the liquid in your cup. In fact, the minimum dipping angle will exceed the recommended angle if you have navigated your cup through a crowded cafeteria. No: surely it is a question of simple timing combined with verticallyheld $(\mathrm{VH})$ dunking. The same formula applies, but the dunk time must be reduced to one quarter of that of an NH biscuit. To prove this, consider that the desired result is a biscuit stabilized by a dry layer, and that the biscuit is now soaked on both sides.

The desired thickness of remaining dry biscuit (whether lateral or central), however, is also related to the transfer time to the oral cavity, as capillary forces will continue to draw liquid from the supersaturated outer layers into the dry layer after withdrawal from the cup. Even a properly dunked VH biscuit, which is considerably more stable than an $\mathrm{NH}$ biscuit (there must be a formula for this), will produce an undesirable mush if the dunking formula ignores cup-to-mouth transfer time.

The problem of 'asymmetrical' glazed biscuits, which have an upper surface notoriously less permeable than the lower, needs further research. The physics gets really complicated with the abhorrent dunking practice observed in Latin countries - applying butter and jam to a croissant and then down into the coffee. Jens C. Jensenius Department of Medical Microbiology and Immunology, University of Aarhus, DK 8000, Aarhus, Denmark 